\title{
BMJ Open Acupuncture for neurogenic bladder due to spinal cord injury: a systematic review protocol
}

\author{
Tao Zhang, ${ }^{1}$ Huilin Liu, ${ }^{1}$ Zhishun Liu, ${ }^{2}$ Linpeng Wang ${ }^{1}$
}

To cite: Zhang $\mathrm{T}$, Liu $\mathrm{H}$, Liu Z, et al. Acupuncture for neurogenic bladder due to spinal cord injury: a systematic review protocol. BMJ Open 2014;4:e006249. doi:10.1136/bmjopen-2014006249

- Prepublication history for this paper is available online. To view these files please visit the journal online (http://dx.doi.org/10.1136/ bmjopen-2014-006249).

Received 29 July 2014 Revised 18 August 2014 Accepted 19 August 2014

CrossMark

${ }^{1}$ Department of Acupuncture and Moxibustion, Beijing Hospital of Traditional Chinese Medicine affiliated to Capital Medical University, Beijing, China

${ }^{2}$ Department of Acupuncture and Moxibustion,

Guang'anmen Hospital, China Academy of Chinese Medical Sciences, Beijing, China

Correspondence to Professor Linpeng Wang; wlp5558@sina.com

\section{ABSTRACT \\ Introduction: Neurogenic bladder is one of the most common complications following spinal cord injury (SCI). In China, acupuncture therapy is a common treatment for neurogenic bladder due to $\mathrm{SCl}$, but its effects and safety remain uncertain. A protocol is described for a systematic review to investigate the beneficial effects and safety of acupuncture for neurogenic bladder due to SCl.}

Methods and analysis: Eight databases will be searched from their inception: the Cochrane Central Register of Controlled Trials (CENTRAL), PubMed, Embase, the China National Knowledge Infrastructure (CNKI), the VIP database, the Wanfang database, the China Doctoral Dissertations Full-text Database (CDFD) and the China Master's Theses Full-text Database (CMFD). Any clinical randomised controlled trials (RCTs) and the first period of randomised cross-over studies related to acupuncture for neurogenic bladder due to $\mathrm{SCl}$ will be included. Outcomes will include change in urinary symptoms, urodynamic tests, clinical assessment and quality of life (QoL). The incidence of adverse events will be assessed as the safety outcome. Study selection, data extraction and quality assessment will be performed independently by two reviewers. Assessment of risk of bias, data synthesis and subgroup analysis will be carried out using Review Manager software.

Ethics and dissemination: Ethics approval is not required as this is a protocol for a systematic review. The findings of this systematic review will be disseminated via peer-reviewed publications and conference presentations.

Trial registration number: PROSPERO (CRD42014010448).

\section{INTRODUCTION}

\section{Description of the condition}

Neurogenic bladder due to spinal cord injury (SCI) refers to bladder dysfunction caused by damage to bladder neural circuits following SCI. It is characterised by lower urinary tract symptoms and is usually followed by complications. ${ }^{1}$ The clinical manifestations of neurogenic bladder dysfunction

\section{Strengths and limitations of this study}

- Study selection, data extraction and quality assessment will be performed by two reviewers independently.

- The authenticity of the included studies will be corroborated by searching relevant published protocols and contacting the first or corresponding author.

- The variety of acupuncture therapies used may make data synthesis and subgroup analysis more difficult.

- Because of the language barrier, Korean and Japanese medical databases will not be covered and some related studies might be missed.

(NBD) include urinary incontinence and urine retention. The underlying pathological mechanisms of NBD symptoms are detrusor overactivity and detrusor-sphincter dyssynergia, depending on the damaged segment of spinal cord and the duration of disease. ${ }^{23}$

Studies have indicated that the incidence of neurogenic bladder is approximately 69$92 \%$ among SCI patients, ${ }^{4-7}$ with annual morbidity varying from 14 to 53 per million population. ${ }^{8}$ It is believed that neurogenic bladder is a major problem, is associated with poor prognosis and is one of the most common complications in SCI patients. ${ }^{4} 1011$ In addition, long-term rehabilitation imposes a heavy economic burden. The US government spends nearly US $\$ 16.3$ billion each year to treat neurogenic bladder. $^{12}$ Therefore, continuous and effective low-cost treatment is needed to improve urinary symptoms and reduce medical costs.

\section{Description of the intervention}

Acupuncture, which has been used for more than 2500 years to cure disease and relieve pain, plays an important role in Traditional Chinese Medicine (TCM) ${ }^{13}$ It is a minimally invasive procedure in which thin metal needles are inserted into specific body points 
and either slowly twisted manually or stimulated electrically. Acupuncture therapy is popular among Chinese people due to its simple operation, low cost and few adverse effects. It is also increasingly practiced and requested by patients and their family members in some western countries. ${ }^{14}$ In 1979 , the WHO drew up a provisional list of 47 diseases that could potentially be treated with acupuncture, including neurogenic bladder due to SCI. ${ }^{15} 16$

\section{How the intervention might work}

Studies have indicated that acupuncture might affect the synthesis and release of neurotransmitters which regulate the physiological function of the bladder, such as substance $\mathrm{P}$, nitric oxide synthase and calcitonin gene-related peptide. ${ }^{17-19}$ In addition, acupuncture could affect discharge frequency controlled by the micturition centres in the central nervous system. ${ }^{20}$ Furthermore, acupuncture could promote the GABAergic system and hinder activation of C-fibres by bladder sensory afferent fibres if needles are inserted at the points near the posterior sacral foramina. ${ }^{21-25}$ In the last 2 years, researchers have begun to focus on the neurorehabilitation and neuroplasticity effects of electroacupuncture for neurogenic bladder due to SCI. ${ }^{26} 27$

\section{Why it is important to do this review}

Currently, acupuncture therapy is a common treatment for neurogenic bladder due to SCI in China. In addition, the number of published intervention studies on the use of acupuncture for this condition has grown markedly over the last few years. Although a systematic review of acupuncture for neurogenic bladder after SCI was published in 2013, the result of the review was inconclusive due to the serious methodological defects of the included studies and the small number of trials assessed in meta-analyses. ${ }^{28}$ It remains uncertain whether acupuncture is effective and safe. Clinical evidence needs to be identified, appraised, graded and summarised for the benefit of both patients and clinicians.

\section{OBJECTIVES}

The objective of this systematic review is to determine whether acupuncture is effective and safe in relieving urinary symptoms, and whether it produces better results in urodynamic tests and improves quality of life (QoL) in patients with neurogenic bladder due to SCI. When possible, both the short-term and the long-term effects will be evaluated.

\section{METHODS}

The methods for this systematic review have been developed according to the recommendations of the Preferred Reporting Items for Systematic Reviews and Meta-Analyses (PRISMA) statement. ${ }^{29}$
Criteria for considering studies for this review

Types of studies

All clinical randomised controlled trials (RCTs) and the first period in randomised cross-over trials will be included in the review, regardless of publication status. Trials will be excluded if they are non-randomised controlled trials, non-randomised cross-over trials, retrospective studies, before-and-after studies or studies that are purely research studies into the mechanisms of acupuncture.

\section{Types of participants}

All participants of any age or gender with stable vital signs, diagnosed with neurogenic bladder caused by SCI, will be included, irrespective of date of injury, its level or its severity. Individuals with neurogenic bladder caused by other diseases such as diabetes mellitus, stroke, tumour, dementia, multiple system atrophy, Parkinson's disease, multiple sclerosis, Guillain-Barre syndrome, spinal canal stenosis, lumbar disc disease or syringomyelia will be excluded. Participants who have undergone a surgical operation for neurogenic bladder will also be excluded.

\section{Types of interventions}

Interventions in the treatment group will include any kind of acupuncture regardless of the site or type of treatment, such as whole body acupuncture, scalp acupuncture, auricular acupuncture, electroacupuncture, fire needling, warm needling, elongated needling, intradermal needling, etc. Acupuncture combined with other conservative treatments will also be included. The control intervention can include no active treatment, sham acupuncture, medication/drugs, rehabilitation (such as bladder training, pelvic-floor muscle exercises, pelvic-floor electro-stimulation and biofeedback), external appliances (condom catheters, pads or penile clamps) or other conservative treatments such as catheterisation (intermittent or indwelling). However, combined interventions consisting of four or more therapies or with potential safety problems will be excluded.

\section{Types of outcome measures}

The primary outcome is the difference in urinary symptoms before and after treatment, as reported by participants in a voiding diary or a self-report questionnaire. It includes the mean number of urination and/or incontinence episodes per $24 \mathrm{~h}$, the number of participants with incontinence or retention, and the number of participants requiring catheterisation.

The secondary outcomes include the following items: (1) changes in urodynamic tests before and after treatment, for example, maximum urinary flow rate (Qmax), postvoiding residual urine volume (RUV) and maximal detrusor pressure; (2) change in clinical assessment before and after treatment, for example, the standardised pad test ( $1 \mathrm{~h}$ or $24 \mathrm{~h}$; quantified leakage), severity of incontinence, and incidence of recurrent urinary 
incontinence or retention; (3) change in a QoL questionnaire before and after treatment, for example, condition-specific QoL measures, general health QoL measures and psychologically related scales; and (4) the effective rate.

Safety outcome measures include the incidence of all reported adverse events such as local pain, haematomas, fainting during acupuncture treatment, and complications related to neurogenic bladder.

\section{Search methods for identification of studies Electronic searches}

The following databases will be searched from their inception, irrespective of publication status: the Cochrane Central Register of Controlled Trials (CENTRAL), PubMed, Embase, the China National Knowledge Infrastructure (CNKI), the VIP database, the Wanfang database, the China Doctoral Dissertations Full-text Database (CDFD) and the China Master's Theses Full-text Database (CMFD).

Studies will also be obtained from the following sources: the reference lists of all included studies or relevant reports of clinical trials or review articles; unpublished conference proceedings relevant to neurogenic bladder due to SCI; and ongoing trials which will be searched through the WHO international clinical trials registry platform (http://apps.who.int/trialsearch/) and Current Controlled Trials (http://www.controlled-trials. com).

\section{Search strategy}

The search will be conducted in English and Chinese using the following terms:

A. Search strategy to locate 'spinal cord injury':

\# 1 spinal cord injury

\# 2 spinal cord trauma

\# 3 spinal cord contusion

\# 4 spinal cord lesion

\# 5 paraplegia

\# 6 or $/ 1-5$

B. Search strategy to locate 'neurogenic bladder':

\# 7 neurogenic bladder

\# 8 bladder disorder

\# 9 uninhibited bladder

\# 10 bladder dysfunction

\# 11 emiction disorder

\# 12 aconuresis

\# 13 incontinence

\# 14 retention

\# 15 uroschesis

\# 16 or/7-15

C. Search strategy to locate acupuncture interventions:

\# 17 acupuncture

\# 18 electroacupuncture

\# 19 fire needle

\# 20 body acupuncture

\# 21 warm needle

\# 22 auricular acupuncture
\# 23 scalp acupuncture

\# 24 elongated needle

\# 25 intradermal needle

\# 26 or $/ 17-25$

\# 276 and 16 and 26

\section{Data collection and analysis}

\section{Selection of studies}

Clinical studies will be identified and included by two independent reviewers ( $\mathrm{TZ}$ and HL) according to the inclusion criteria. The two reviewers will read the titles, abstracts and full texts if necessary, and collect the studies meeting the inclusion criteria. The authenticity of the collected studies will be checked directly by searching related published protocols and contacting the first or corresponding author. If telephone numbers are not available, email or post will be used. Suitable RCTs will be selected and cross-checked by the two reviewers (TZ and HL). Any disagreement will be discussed after cross-checking and adjudicated by the third reviewer (LW). The process of studies selection is presented in a PRISMA flow diagram (figure 1).

\section{Data extraction and management}

Two independent researchers (TZ and HL) will extract the data from the included studies into an extraction form developed according to recommendations in the Cochrane Handbook. The extraction form consists of 10 major items: general information (author, working location, publication date, journal, etc), the participants, characteristics of interventions, outcomes, randomisation, allocation concealment, incomplete data, blinding, selective report, and conflicts of interest. The result of data extraction will also be cross-checked. Any disagreement will be discussed and adjudicated by a third reviewer (LW).

\section{Assessment of risk of bias in included studies}

The risk of bias will be evaluated by two reviewers independently. The result of the assessment will also be cross-checked. Any disagreement will be discussed and adjudicated by a third reviewer. According to the methods for assessing risk of bias using the 'Risk of bias' tool of the Cochrane Handbook (V.5.1.0), the studies will be categorised as 'high risk' of bias, 'low risk' of bias, or 'unclear risk' of bias. The following types of bias will be assessed: (1) selection bias: random sequence generation and allocation concealment; (2) performance bias: blinding of investigators, participants and care providers; (3) detection bias: blinding of outcome assessment; (4) attrition bias: incomplete data/differential dropout; (5) reporting bias: selective reporting; (6) other bias: for example, conflict of interest, follow-up, non-intention-to-treat or per-protocol analysis, etc.

Measures of treatment effect

For dichotomous data, the results of each study will be pooled and presented as a risk ratio with $95 \%$ CIs. A 


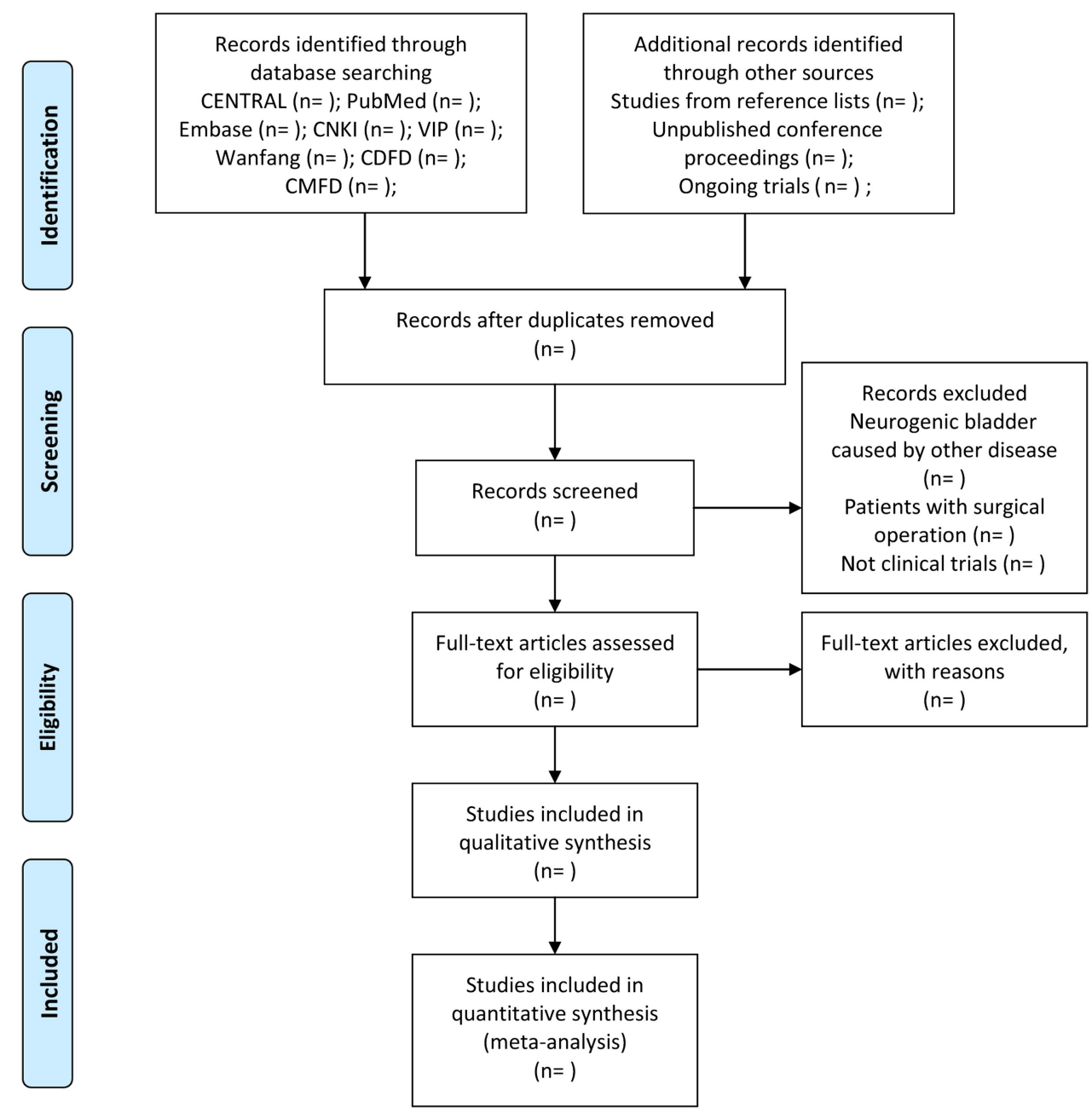

Figure 1 Flow diagram of the trial selection process.

mean difference with $95 \%$ CI will be used for continuous data.

\section{Dealing with missing data}

If required data are ambiguous or not reported in the clinical articles, reviewers will contact the first or corresponding author of the studies by telephone, email or post and collect the missing data using the data extraction form.

\section{Assessment of heterogeneity}

The heterogeneity of the studies will be assessed before meta-analysis based on clinical similarities in populations and interventions. Clinical and methodological heterogeneity will be judged according to the data recorded on the extraction form. Statistical heterogeneity will be calculated by using the Mantel-Haenszel $\chi^{2}$ test for heterogeneity. A result with a $\mathrm{p}$ value $<0.10$ and a high $\mathrm{I}^{2}$ value indicates statistically significant heterogeneity. According to the Cochrane Handbook, $\mathrm{I}^{2}$ values can be classified into four categories: a value of $0-40 \%$ indicates little or no heterogeneity; a value of $30-60 \%$ indicates moderate heterogeneity; a value of $50-90 \%$ indicates substantial heterogeneity; and a value of $75-100 \%$ indicates considerable heterogeneity. Statistical heterogeneity also depends on the magnitude of effects, the direction of results and the strength of evidence.

\section{Assessment of reporting biases}

Reviewers will search for the published protocols of the trials or contact the first or corresponding author of the study to acquire information regarding selective reporting. The result will be judged as having a 'high risk' of bias, a 'low risk' of bias or an 'unclear risk' of bias according to the criteria of the 'Risk of bias' assessment tool of the Cochrane Handbook (V.5.1.0). Funnel plots will be generated to analyse the potential publication bias if more than 10 trials are included.

\section{Data synthesis}

Data synthesis will be carried out using Review Manager (V.5.2) statistical software provided by the Cochrane Collaboration. If two or more eligible studies with low clinical, methodological and statistical heterogeneity are identified, the studies will be combined for meta-analysis. We will consider using the random-effects 
model during the meta-analysis. For the cross-over studies, only data from the period before the first crossover will be analysed.

\section{Subgroup analysis}

If a sufficient number of randomised trials are identified, subgroup analyses will be performed according to: (1) different types of acupuncture therapy; (2) neurological level of SCI (low vs high); (3) treatment duration (eg, less than or more than 2 weeks); and (4) clinical manifestation of neurogenic bladder (incontinence or retention).

\section{Sensitivity analysis}

Sensitivity analysis will be carried out when significant heterogeneity still exists after subgroup analysis and when no errors are found in the data input steps. The meta-analysis will be repeated after lower quality studies are excluded. The results of these two meta-analyses will then be compared. It will be decided by discussion whether the lower quality studies will be excluded or not, depending on their sample size, strength of evidence and influence on the pooled effect size.

\section{DISCUSSION}

This systematic review will provide the latest analysis of the current state of acupuncture for neurogenic bladder due to SCI. It may provide benefits and clinical evidence for both patients and clinicians.

Although a related systematic review has been published by Hao and colleagues, it still remains uncertain whether acupuncture is an effective and safe therapy for neurogenic bladder due to SCI. ${ }^{28}$ In that review, the deadline for study retrieval and selection was September 2012. Only eight studies were selected and there was no verification process (five of the studies were included in meta-analyses). The outcome measures only consisted of urodynamic tests and effective rate. The diversity, validity and reliability of the measures made it difficult to conduct meta-analyses. In addition, the included studies had significant heterogeneity. As a result, no definite conclusion could be reached because of the design defects of the included studies and the small number of studies analysed in the meta-analyses. Moreover, the included studies provided no data which could be used for assessing safety. Therefore, there are some major differences between the review of Hao et al and our planned systematic review. First, the number of published RCTs of acupuncture for neurogenic bladder due to SCI has increased since 2012. More studies may be included in this systematic review. Second, following the statement of the International Committee of Medical Journal Editors (ICMJE) published in 2004, which requires that all clinical trials be registered in order to improve the transparency and credibility of clinical evidence, ${ }^{30}$ the authenticity of each included study will be checked by searching relevant published protocols and contacting the first or corresponding author. Despite recent increased publicity, some researchers still ignore the importance of trial registration, especially in China. Therefore, it is necessary to verify the authenticity of included studies in order to reduce bias. Third, the outcome measures of our planned review will include differences in urodynamic tests, but will also focus on changes in urinary symptoms, in clinical assessment and in patients' QoL. In addition, the incidence of adverse events will also be assessed as a safety outcome.

Nevertheless, this systematic review will still have some limitations. As an important branch of TCM, acupuncture therapy is classified and denominated according to the type of manipulation and needling instrument. As a result, interventions vary greatly, which increases the difficulty of subgroup analysis. A large number of subgroups will reduce the comparability of studies and increase the complexity of meta-analysis, which may in turn lead to the wrong conclusion. In addition, studies will be obtained from the databases list in the protocol. Because of the language barrier, Korean and Japanese medical databases will not be covered and some relevant studies might be missed.

Acknowledgements We would like to acknowledge the following for their contribution to the protocol: Dr Ines Eisner-Janowicz, who is a special researcher in Beijing Hospital of Traditional Chinese Medicine affiliated to Capital Medical University, Beijing, China, helped us to revise the English of the manuscript; and editors of the Cochrane Incontinence Group who gave us methodological advice and support.

Collaborator Ines Eisner-Janowicz.

Contributors HL: planned the protocol; LW: assisted in protocol design; ZL, LW: provided clinical advice on the study protocol; ZL: devised the search strategy; TZ, HL: drafted the protocol; TZ, HL: will search for studies, and extract and analyse data.

Funding This work is supported by the following funding: the Major Scientific and Technological Research Projects of Beijing (Project Number: D09050703550902), China; The 'Twelfth Five-Year Plan' of the National Science and Technology Support Program (Project Number: 2013BAl13B03), China; the special project for the national clinical research bases construction of traditional Chinese medicine belonging to the State Administration of Traditional Chinese Medicine of the People's Republic of China (Project Number: JD2X2012152), China.

Competing interests None.

Provenance and peer review Not commissioned; internally peer reviewed.

Data sharing statement The findings of this systematic review will be disseminated via peer-reviewed publications and conference presentations. All of the data will be available.

Open Access This is an Open Access article distributed in accordance with the Creative Commons Attribution Non Commercial (CC BY-NC 4.0) license, which permits others to distribute, remix, adapt, build upon this work noncommercially, and license their derivative works on different terms, provided the original work is properly cited and the use is non-commercial. See: http:// creativecommons.org/licenses/by-nc/4.0/

\section{REFERENCES}

1. Stöhrer M, Goepel M, Kondo A, et al. The standardization of terminology in neurogenic lower urinary tract dysfunction with suggestions for diagnostic procedures. Neurourol Urodyn 1999;18:139-58. 
2. Weld KJ, Dmochowski RR. Association of level of injury and bladder behavior in patients with post-traumatic spinal cord injury. Urology 2000;55:490-4.

3. Chua HC, Tow A, Tan ES. The neurogenic bladder in spinal cord injury-pattern and management. Ann Acad Med Singapore 1996;25:553-7.

4. Dahlberg A, Perttilä I, Wuokko E, et al. Bladder management of persons with spinal cord lesion. Spinal Cord 2004;42:694-8.

5. Hansen RB, Biering-Sørensen F, Kristensen JK. Bladder emptying over a period of 10-45 years after a traumatic spinal cord injury. Spinal Cord 2004;42:631-7.

6. Patki $\mathrm{P}$, Woodhouse J, Hamid R, et al. Lower urinary tract dysfunction in ambulatory patients with incomplete spinal cord injury. J Urol 2006;175:1784-7.

7. Podnar S, Trsinar B, Vodusek DB. Bladder dysfunction in patients with cauda equina lesions. Neurourol Urodyn 2006;25:23-31.

8. Schoenfeld AJ, McCriskin B, Hsiao M, et al. Incidence and epidemiology of spinal cord injury within a closed American population: the United States military (2000-2009). Spinal Cord 2011;49:874-9.

9. Pirouzmand F. Epidemiological trends of spine and spinal cord injuries in the largest Canadian adult trauma center from 1986 to 2006. J Neurosurg Spine 2010;12:131-40.

10. Cruz CD, Cruz F. Spinal cord injury and bladder dysfunction: new ideas about an old problem. Sci World J 2011;11:214-34.

11. Cheng PT, Wong MK, Chang PL. A therapeutic trial of acupuncture in neurogenic bladder of spinal cord injured patients: a preliminary report. Spinal Cord 1998;36:476-80.

12. Wilson L, Brown JS, Shin GP, et al. Annual direct cost of urinary incontinence. Am J Obstet Gynecol 2001;98:398-406.

13. Lee MHM, Liao SJ. Acupuncture in psychiatry. In: Kottke FJ Lehmann JF, eds. Krusen's Handbook of Physical Medicine and Rehabilitation. 4th edn. Philadelphia, PA: WB Saunders, 1990:402-9.

14. NIH Consensus Conference. Acupuncture. JAMA 1998;280:1518-24.

15. Bonnerman R. Acupuncture: the World Health Organization view. World Health, 1979

16. World Health Organization: Geneva. Acupuncture: review and analysis of reports on controlled clinical trials. WHO Library Cataloguing-in-Publication Data 2003;26.

17. Wang XY, Zheng HT, Wang SY, et al. Afferent nerve mechanism of electro-acupuncturing Zhonglushu (BL29) inhibiting bladder overactivity. Shanghai J Acupunct Moxibust 2003;22:17-20.
18. Chen $Y L$, Cen J, Hou WG, et al. Effects of electroacupuncture treatment on nitrergic neurotransmitter in bladder neck and detrusor of rats with unstable bladder. J Chin Integr Med 2006;4:73-5.

19. Sun W, Yang F, Qi Q. Urodynamic effects of electroacupuncture at Ciliao on overactive bladder in rats and CGRP, SP expressions of dorsal horn of spinal cord. J Dalian Medical Univ 2012;34:220-4.

20. Wang H, Tanaka Y, Kawauchi A, et al. Acupuncture of the sacral vertebrae suppresses bladder activity and bladder activity-related neurons in the brainstem micturition center. Neurosci Res 2012;72:43-9.

21. Hino $\mathrm{K}$, Honjo $\mathrm{H}$, Nakao $\mathrm{M}$, et al. The effects of sacral acupuncture on acetic acid-induced bladder irritation in conscious rats. Urology 2010;75:730-4

22. Yu JN, Ma XJ, Liu ZS, et al. Effect of electroacupuncture at "Ciliao"(BL 32) on c-fos expression in the sacral segment of spinal cord in rats with detrusor hyperreflexia. Zhen $\mathrm{Ci}$ Yan Jiu 2010;35:204-7, 221.

23. Ma X, Yu J, Liu Z, et al. Effect of electroacupuncture at "BL 32" on VR1 expression in the sacral micturition center of rats with detrusor hyperreflexia. J Liaoning Univ TCM 2010;12:198-201.

24. Wang Y, Liu B, Liu Z. The specific effect and afferent mechanism of electroacupuncture at BL32 for overactive bladder. [Doctoral dissertation]. China Academy of Chinese Medical Sciences, 2012.

25. Wang $\mathrm{H}$, Tanaka $\mathrm{Y}$, Seki $\mathrm{H}$, et al. Acupuncture stimulation to the sacral segment affects state of vigilance in rats. Neurosci Res 2007;57:531-7.

26. $\mathrm{Li} \mathrm{J}$, Zhang $\mathrm{H}$. The effect on electrical acupuncture of rats with neurogenical bladder (urinary retention) after sacral spinal cord injured through NGF-TrkA and PI3-K/Akt pathway [Master's thesis] Hunan University of Chinese Medicine, China, 2013.

27. Yan Q, Ruan JW, Ding Y, et al. Electro-acupuncture promotes differentiation of mesenchymal stem cells, regeneration of nerve fibers and partial functional recovery after spinal cord injury. Exp Toxicol Pathol 2011;63:151-6.

28. Hao C, Wu F, Guo Y, et al. Acupuncture for neurogenic bladder after spinal cord injury: a systematic review and meta-analysis. Eur $J$ Integr Med 2013;5:100-8.

29. Moher D, Liberati A, Tetzlaff J, et al. Preferred reporting items for systematic reviews and meta-analyses: the PRISMA statement. BMJ 2009;339:b2535.

30. De Angelis C, Drazen JM, Frizelle FA, et al. International Committee of Medical Journal Editors. Clinical trial registration: a statement from the International Committee of Medical Journal. N Engl J Med 2004;351:1250-1. 